\author{
Mahendra Narain Mishra ${ }^{1 \star}$ and \\ Vandana $\mathrm{Lal}^{2}$ \\ ${ }^{1} \mathrm{MD}$, Pathology, ESHI Diploma, UK \\ ${ }^{2}$ National Reference Laboratory, Dr Lal Path Labs, \\ India
}

Dates: Received: 05 November, 2016; Accepted: 29 November, 2016; Published: 30 November, 2016

*Corresponding author: Dr. (Col) Mahendra Narain Mishra, MD Pathology, ESHI Diploma, 1st Floor,

NIBTS Building, Lisburn Road, Belfast City Hospital, Belfast, UK, BT9 6LS, Tel: +442895046151; E-mail: mnmishra@hotmail.com

www.peertechz.com

Keywords: Luminex; Antibody; College of american pathologists; External proficiency test

\author{
Research article
}

\section{College of American Pathologists EQAS for Luminex Antibody Test in a Stand -Alone Accredited Indian Laboratory}

\section{Introduction}

In order to maintain confidence in reporting for laboratory accreditation, it is imperative for laboratories to participate in external proficiency testing (EPT) for all tests offered by the laboratory. Luminex based assays are being used worldwide for semiquantitative measurement of antibodies in sera of potential allograft recipients and have been used for the formulation of guidelines of the British Society of Histocompatibility and Immunogenetics for risk stratification of solid organ recipients [1]. Over the last five years Luminex antibody testing, especially donor specific IgG antibody (DSA) which is a Luminex crossmatch has become very popular in India and most centers are using it in lieu of Single antigen bead testing which is expensive and may be give many false positive results due to its exquisite sensitivity [2]. This test detects only anti HLA - A $B$ and DRB1 IgG antibodies with lower sensitivity and specificity than Single antigen beads [3]. Panel reactive screen (pooled bead assay) and to a lesser extent antibody specification (phenotype bead assay) are being increasingly used in the Indian subcontinent for detection of anti HLA antibodies. No external proficiency testing (EPT) survey is available in India for from the providers of the kit for Luminex antibody detection. The College of American Pathologists (CAP) provides a survey that does not grade antibody tests done on Luminex platform due to low number of participants for this assay, and the results are compared with those obtained on flowcytometry. We analysed our one year's CAP EPT performance for the year 2014 with the aim of evaluating the adequacy of the CAP samples for antibody detection by Luminex.

\section{Materials and Methods}

Three CAP EPT surveys (MX-1 A, B, C) for HLA class I and (MX- 2 A, B and C) for HLA class II, comprising of four samples for HLA - class I, two samples for HLA - class II were processed during the period January - December 2014. Lifecodes Screen \& identification kits were used for antibody screening and specification. Lifecodes DSA kits were used for Luminex crossmatch. All kits were purchased from Immucor, India. The protocol mentioned in the product insert was followed for performing the tests and $\mathrm{MATCH}$ ITI Antibody software was used for analysis. All EQAS samples were stored as per specification (2-80 Celsius) until processing which was done within $72 \mathrm{hrs}$ of receipt. A positive or negative assignment was made on the basis of both mean fluorescence intensity (MFI) and score which incorporates a correction for the background. MFI $>500$ was assigned positive only if the score was $\geq$ two. This was followed for all three antibody detection assays. The results obtained were compared with that of Flowcytometry results provided in CAP survey for concordance. Flowcytometry identified more antibodies than Luminex for all samples and in one class I sample specification was different from Luminex although Luminex is known to be more sensitive [2]. Anti DQA1 and anti DPB1 antibodies were not included in this analysis as the kit is known not to detect them. Higher PRA of sample was associated with greater number of antibodies and a concomitant increase in specificities not identified by Luminex. Performance in EQAS was satisfactory because results for first 16 
specificities which are considered for evaluation showed concordance. A summary of the results is shown in Table 1 . The results of antibody specification are depicted in Table 2 including the percentage PRA for all samples. Inter - laboratory comparison (ILC) was performed for two samples (one negative and one positive) for all surveys for which discordant results were obtained but ILC results were concordant for both positive and negative samples.

\section{Discussion}

Antibody screening may be performed on the sample as the results were entirely concordant by both methods as expected for a screening test. CAP result format for antibody specification has a column of antibody specificities and another for additional specificities although only 16 antibodies are to be reported. Antibody specificities were matched for all positive class I samples with few exceptions. A higher

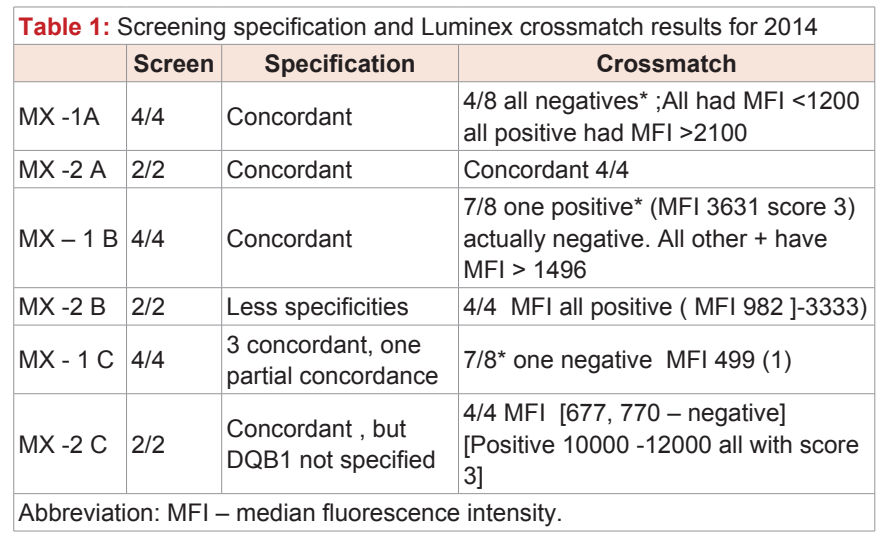

Table 2: showing the results of antibody specification by Flowcytometry and Luminex.

\begin{tabular}{|c|c|c|}
\hline Survey ID & $\begin{array}{l}\text { Number of specificities } \\
\text { and (\% PRA) }\end{array}$ & $\begin{array}{l}\text { Extent of match and unidentified } \\
\text { antibodies }\end{array}$ \\
\hline$M X-101 P$ & n 27 (92) & Entirely matched \\
\hline 02 & n $18(98)$ & Entirely matched \\
\hline 03 & n $32(84)$ & Entirely matched \\
\hline 04 & n $28(80)$ & Entirely matched \\
\hline \multicolumn{3}{|l|}{$M X-2 A$} \\
\hline $01-P$ & n 8 (73) & DR8 (on same beads as assigned) \\
\hline $02-P$ & n 8 (70) & Entirely matched \\
\hline MX -1B 1-07 P & n $45(80)$ & A 43, Cw6 \\
\hline $1-08 \mathrm{P}$ & n $16(60)$ & B48 \\
\hline 1-09 P & n 27 (94) & A3,B13, Cw2,Cw9 \\
\hline $1-10 P$ & n $22(90)$ & B76 \\
\hline MX 2B 5 P & n $6 \quad(57)$ & DR51 not detected \\
\hline $6 \mathrm{P}$ & n 20 (57) & $\begin{array}{l}\text { DQ2,DQ4,DQ5,DQ6,DQ9,DQ7,DR52, } \\
\text { DR14, DR12, DR15 }\end{array}$ \\
\hline MX 1 C $-13 P$ & n $7(60)$ & Entirely matched \\
\hline $14 \mathrm{P}$ & $n=14(48)$ & $\begin{array}{l}\text { B41 detected but negative in results } \\
\text { Additional specifications on Flow- } \\
\text { A43,A11, B35 not on }\end{array}$ \\
\hline $15 \mathrm{P}$ & $n=50(80)$ & Cw 6 detected but negative in results \\
\hline$-16 P$ & $\mathrm{n}=0 \%$ & Negative \\
\hline $\mathrm{MX} 2 \mathrm{C}-09-\mathrm{P}$ & $n=8(67)$ & $\begin{array}{l}\text { DR4, DR7, DR12 (all are on the true } \\
\text { positive beads) }\end{array}$ \\
\hline$-10 P$ & & Negative \\
\hline
\end{tabular}

degree of concordance was observed if PRA was higher. The HLA class I IgG antibodies not detected included A43, B41, B48, B76 (two samples), Cw2 (two samples) and Cw9 - all of which are relatively infrequent in Indian population. However these antibodies had been identified in other samples. Antibody specification by tailed analysis for class II did not assign some DQB1 specificities. Phenotype beads were coated with DRB1, DQB1, DPB1 and DR51/52/53 antigens but only the DQB1 antigens were not assigned. It may be useful to improve the software further to enable detection of DQA1 and DPB1 antibodies which are present on all HLA - Class II screen and phenotype beads but cannot be identified. Other helpful measure will be, add more beads and probably change the combination of antigens coated onto the beads to improve antibody specification but with phenotype assay, the level of specification can never be same as that of Single antigen bead assay.

Lifecodes DSA kit contains both HLA class I and II capture molecules, unlike Flowcytometry, in which each sample of the survey is tested for either class I or class II reactivity. Discrepant results were seen only for $\mathrm{T}$ cell crossmatch. This could be due to matrix interference as the class I and II capture molecules cannot be separated for testing enriched $\mathrm{T}$ cells provided. Raw data revealed that there was class II reactivity in avll these samples. Four of the six discrepant results were incorrectly assigned as negative although the MFI was higher than 500 but score was less than 2. The fifth sample had MFI of 499 with score of zero and was assigned as negative. Normally score is accorded a priority over MFI for determining a value as positive or negative because score incorporates for background correction. There is no explanation for the only negative class I crossmatch which was assigned incorrectly as positive (Table 1).

\section{Conclusion}

CAP survey may be used for antibody screening and specification on Luminex platform, but Luminex crossmatch will require another dedicated survey for evaluation. The performance of the kits can probably be improved by using additional beads and also improving the software. Antibody specification will require a closer scrutiny of results to assign the other specificities of DQB1 especially if it is positive for them along with the DRB1 assigned.

\section{Acknowledgement}

I am grateful to our technicians Ms Asha Amoli, Ms Anchal Thakur Arora and Mr Rakesh Kumar Jha for processing the samples

\section{References}

1. Guidelines for the detection and characterization of clinically relevant antibodies in allotransplantation.

2. Gombos P, Opelz G, Scherer S, Morath C, Zeier M, et al. (2013) Influence of Test Technique on Sensitization Status of Patients on the Kidney Transplant Waiting List. Am J of Transplant 13: 2075-82.

3. Caro-Oleas JL, Gonzalez-Escribano MF, Toro-Llamas M, Martinez-Bravo MJ, Aguilera I, et al. (2010) Donor-specific antibody detection: comparison of single antigen assay and Luminex crossmatches. Tissue Antigens 76: 398403.

4. Riethm u“ller S, Ferrari-Lacraz S, Mu“ller MK, Raptis DA, Hadaya K, et al. (2010) Donor-Specific Antibody Levels and Three Generations of Crossmatches to Predict Antibody Mediated Rejection in Kidney Transplantation. Transplantation 7: 90: 160-7.

5. Tait BD, Süsal C, Gebel HM, Nickerson PW, Zachary AA et al. (2013) Consensus guidelines on the testing and clinical management issues associated with HLA and non-HLA anti- bodies in transplantation. Transplantation 95: 19-47.

Copyright: (c) 2016 Mishra MN, et al. This is an open-access article distributed under the terms of the Creative Commons Attribution License, which permits unrestricted use, distribution, and reproduction in any medium, provided the original author and source are credited. 